\title{
Intrinsic Shape of Bulges in Disk Galaxies
}

\author{
J. Mendez-Abreu ${ }^{1}$, J. A. L. Aguerri ${ }^{2}$, E. M. Corsini ${ }^{3}$, \\ and E. Simonneau ${ }^{4}$ \\ ${ }^{1}$ INAF-Osservatorio Astronomico Padova, Italy email: jairo.mendez@oapd.inaf.it \\ ${ }^{2}$ Instituto Astrofisica Canarias, Spain \\ ${ }^{3}$ Dipartimento Astronomia. Universita di Padova, Italy \\ ${ }^{4}$ Institut d'Astrophysique de Paris, France
}

\begin{abstract}
We analyzed the 2MASS images of a sample of 148 bright, nearby and unbarred galaxies to derive the photometric parameters of their bulge and disk by applying an accurate 2D decomposition algorithm. As a model for the bulge we have considered a system where the surfaces of equal density of light are coaxial and concentric ellipsoids with independent values of the principal axes $\mathrm{A}>\mathrm{B}>\mathrm{C}$. If the emitting surfaces are concentric, coaxial but non-homologous ellipsoids, their projection on the plane of the sky will be concentric, nonhomologous and non-coaxial ellipses (Stark 1977). Therefore if we identify the projected ellipses with the isophotal contours of a bulge, the observed twist between the position angle of the bulge and disk components can be understood as a hint for triaxiality.

Following the procedure described by Simonneau et al. (1998), we are able to express the intrinsic semi-axis values (A, B, C) of the triaxial ellipsoid as a function of the semi-axis measured on to its projected ellipse $(\mathrm{a}, \mathrm{b})$, position angle of the ellipse $(\delta)$, and angles which define the line of sight with respect to the ellipsoid $(\theta, \phi)$.

However, since the $\phi$ angle is observationally undetermined, the intrinsic shape of bulges is still an ambiguous problem. Thus, it is customary to resort to a statistical approach for deducing the probability distribution function (PDF) of the intrinsic axis ratio of bulges. Since we have already inverted the problem, putting the intrinsic variables as a function of the measured variables $(\mathrm{E}, \delta)$ and the variables $(\theta, \phi)$ which are defined in a probabilistic way, and we have assumed that they are independent, the PDF can be determined simply by the multiplication of the integrated single probabilities of these variables.

We have found that integrating the derived PDF over the different ellipsoid regions (prolate, triaxial and oblate), $\sim 45 \%$ of bulges have a triaxial structure, about $26 \%$ are oblate ellipsoids, $23 \%$ are prolate ellipsoids and the remaining $5 \%$ are spherical.

A typical bulge from our PDF, calculated as the mean value of the axis ratios of the ellipsoids weighted with the $\mathrm{PDF}$, have axis ratios $\langle\mathrm{P}\rangle=1.66$ and $\langle\mathrm{Q}\rangle=1.34$. This means that the axis ratio in the equatorial plane of the galaxy is $\mathrm{B} / \mathrm{A}=0.81$, the ratio between the major and the polar axis is $\mathrm{C} / \mathrm{A}=0.60$ and the triaxiality parameter is $\mathrm{T}=0.55$, which is in good agreement with the previous results by Bertola et al. (1991).

We also found a correlation between the Sérsic index and the intrinsic flattening of the bulges. We observed that bulges tend to be less flattened $(\mathrm{C} / \mathrm{A}=0.64)$ in more-concentrated galaxies $(\mathrm{n}>2)$ and more flattened $(\mathrm{C} / \mathrm{A}=0.54)$ in less-concentrated galaxies.
\end{abstract}

Keywords. galaxies: bulges, galaxies: photometry, galaxies: structure

\section{References}

Bertola, F., Vietri, M. \& Zeilinger, W. W. 1991,ApJ, 374, 13.

Simonneau, E., Varela, A. M. \& Muñoz-Tuñón, C. 1998, Il nuovo cimento, 113B, 927.

Stark, A. 1977, ApJ, 213, 368. 